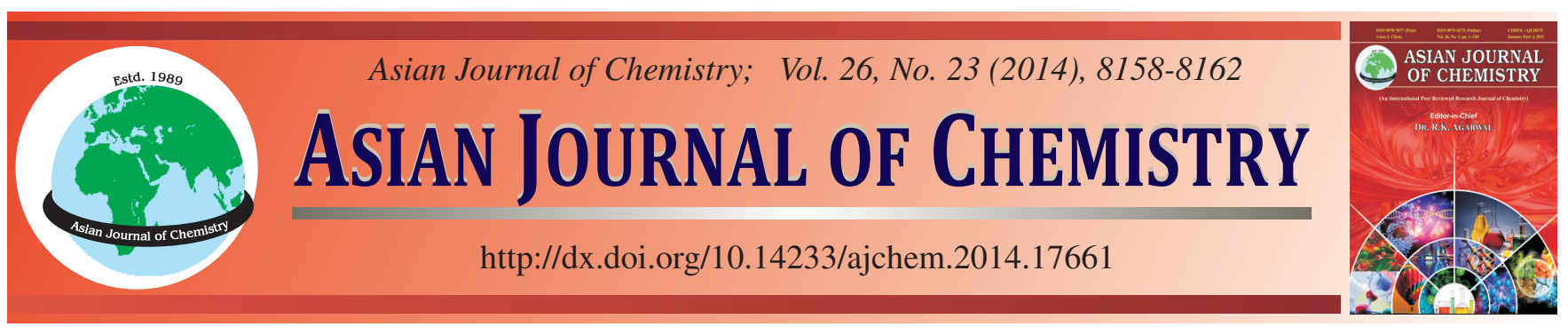

\title{
Development and Validation of Process for Resolution of Praziquantel Amine for Preparation of Chiral Praziquantel
}

Changyan Hu, Dongling Wang, Kunquan Chen, Xu Liu, Qichao Chen and Dequn Sun*

Marine College, Shandong University, Weihai Wenhua West Road, No. 180, Weihai 264209, P.R. China

*Corresponding author: E-mail: dequn.sun@sdu.edu.cn

Received: 25 March 2014;

Accepted: 21 May 2014;

Published online: 15 November 2014;

AJC-16316

\begin{abstract}
Investigation of the viability of scale up preparation for chiral praziquantel was included in WHO/TDR business plan. Resolution is the major strategy for preparation of single enantiomer. In this study, a stable and effective resolution approach toward the important intermediate praziquantel amine for preparation of chiral praziquantel was developed and the process was optimized to afford high $e e$ value and good yield. The suitable HPLC method was validated for the determination of $e e$ value of chiral praziquantel amine.
\end{abstract}

Keywords: Schistosomiasis, Praziquantel, Resolution, Chiral praziquantel amine, Chiral HPLC analysis, Validation.

\section{INTRODUCTION}

Schistosomiasis is one of the most burdensome and neglected tropical diseases, accounting for an extraordinarily high level of suffering around the world ${ }^{1}$. The World Health Organisation estimates 200 million people are infected and it is calculated that further 600 million people are at risk of infection.

So far there is only one drug of choice, a highly effective small molecule called praziquantel (PZQ) ${ }^{2}$. However, the drug is currently manufactured and administered as a racemate $(1: 1$ mixture of enantiomers). The L-(-)-enantiomer is the eutomer ${ }^{3-5}$ and has the $(\mathrm{R})$ configuration $^{6}$, which resulted in fewer side effects than the racemate ${ }^{4}$. The inactive $(+)$-enantiomer is associated with side effects and is also primarily responsible for the extremely bitter taste of the pill ${ }^{7}$; There has been much recent debate whether praziquantel, like so many other drugs, is destined to become less useful because of drug-resistance ${ }^{8-10}$ and several strands of evidence indicate that it may not escape this fate. Therefore, WHO established a business plan to investigate the possibility to switch chiral praziquantel into market ${ }^{11}$. Todd et al. ${ }^{12}$ has discovered first time that using resolution approach to achieve rac-praziquantel with $77 \%$ yield and high ee value. However, can such enantiopure praziquantel be obtained without a significant increase in price compared with rac-praziquantel? It is a challenge for either academia or industry to solve this problem. In order to reduce the pro-duction price, the efficiency of resolving process needs improvement and validation to match industrial production. Furthermore, R- and S-praziquantel would be valuable molecule to elucidate the action mechanism of praziquantel, which is still not clear after use of praziquantel for 30 years. Therefore, developing the stable and practical strategy to produce the qualified chiral praziquantel is highly desirable. This article described herein an effective resolution process and new devitrification method to obtain high yield and high quality of chiral praziquantel amine and also validated a simple and sensitive HPLC method for measure of $e e$ value of chiral praziquantel amine.

\section{EXPERIMENTAL}

HPLC analysis of rac-praziquantel amine: The racPraziquantel amine could be baseline separated on a AD-H $(4.6 \times$ $250 \mathrm{~mm}$, DAICEL) column with hexane:ethanol:triethylamine (80:20:0.1) as eluent, flow rate $1 \mathrm{~mL} \mathrm{~min}^{-1}, \mathrm{t}_{\mathrm{R}}=21.3 \mathrm{~min}, \mathrm{t}_{\mathrm{S}}=$ $29.4 \mathrm{~min}$.

Resolution of praziquantel amine for rac-praziquantel amine

Without seeding: rac-Praziquanamine ( $1 \mathrm{~g}, 4.95 \mathrm{mmol})$ and (-)-dibenzoyl-L-tartaric acid (1.772 g, $4.95 \mathrm{mmol})$ were dissolved in ethanol $(24 \mathrm{~mL})$ and water $(30 \mathrm{~mL})$ by heating the stirred mixture to $76^{\circ} \mathrm{C}$ for $4 \mathrm{~h}$. Then the solution was put into $10^{\circ} \mathrm{C}$ refrigerator immediately for $3 \mathrm{~h}$. The crystals were filtered to give $r a c$-praziquantelamine-L-tartaric acid $1.356 \mathrm{~g}$ (yield of salt $48.9 \%$, after liberation, ee value of rac-praziquantelamine was $76 \%$ determined by HPLC.

With seeding: rac-Praziquanamine $(1 \mathrm{~g}, 4.95 \mathrm{mmol})$ and (-)-dibenzoyl-L-tartaric acid (1.772 g, $4.95 \mathrm{mmol})$ were dissolved in ethanol $(24 \mathrm{~mL})$ and water $(30 \mathrm{~mL})$ by heating 
the stirred mixture to $76^{\circ} \mathrm{C}$ for $4 \mathrm{~h}$. The mixture was added $5 \mathrm{mg}$ S-praziquantel amine-D-tartaric acid and put into $10^{\circ} \mathrm{C}$ refrigerator immediately for $3 \mathrm{~h}$. The crystals were filtered to give rac-praziquantel amine-L-tartaric acid $1.385 \mathrm{~g}$ (yield of R-salt $50.0 \%$, after liberation, ee value of rac-praziquantel amine was $82 \%$ ee determined by HPLC.

Recrystallization of salt: The salt rac-praziquantel amine-L-tartaric acid $\left(0.5 \mathrm{~g}\right.$ salt, $[\alpha]_{\mathrm{D}}{ }^{20}=-132.9,78 \%$ ee $)$ was dissolved in ethanol/isopropanol/water $(1: 1: 2$, total volume $=$ $6 \mathrm{~mL}$ ) by heating to $76^{\circ} \mathrm{C}$ and then the solution was put into $10^{\circ} \mathrm{C}$ refrigerator for $12 \mathrm{~h}$. The crystals were filtered and dried to yield the salt as white crystals $0.410 \mathrm{~g},[\alpha]_{\mathrm{D}}{ }^{20}=-155.9$ (determined by polarimetry, $\mathrm{C}=1, \mathrm{CH}_{3} \mathrm{OH}$ ), $99 \%$ ee .

\section{Resolution of praziquantel amine for $\mathrm{S}$-praziquantel amine}

Without seeding: rac-Praziquanamine ( $1 \mathrm{~g}, 4.95 \mathrm{mmol})$ and (-)-dibenzoyl-D-tartaric acid (1.772 g, $4.95 \mathrm{mmol})$ were dissolved in ethanol $(24 \mathrm{~mL})$ and water $(30 \mathrm{~mL})$ by heating the stirred mixture to $76^{\circ} \mathrm{C}$ for $4 \mathrm{~h}$. The solution was put into $10{ }^{\circ} \mathrm{C}$ refrigerator immediately for $3 \mathrm{~h}$. The crystals were filtered to give S-praziquantel amine-D-tartaric acid $0.928 \mathrm{~g}$ (yield of salt $37.3 \%$, after liberation, ee value of S-praziquantel amine was $94 \%$ determined by HPLC.

With seeding: rac-Praziquanamine $(1 \mathrm{~g}, 4.95 \mathrm{mmol})$ and (-)-dibenzoyl-D-tartaric acid (1.772 g, $4.95 \mathrm{mmol})$ were dissolved in ethanol $(24 \mathrm{~mL})$ and water $(30 \mathrm{~mL})$ by heating the stirred mixture to $76{ }^{\circ} \mathrm{C}$ for $4 \mathrm{~h}$. The mixture was added $5 \mathrm{mg} \mathrm{S}$ praziquantel amine-D-tartaric acid and put into $10{ }^{\circ} \mathrm{C}$ refrigerator immediately for $4 \mathrm{~h}$. The crystals were filtered to give $\mathrm{S}-$ praziquantel amine-D-tartaric acid $1.210 \mathrm{~g}$ (yield of salt $48.7 \%$, after liberation, $e e$ value of S-praziquantel amine was $94 \%$ determined by HPLC.

Recrystallization of salt: The salt S-praziquantel amineD-tartaric acid $\left(0.5 \mathrm{~g},[\alpha]_{\mathrm{D}}{ }^{20}=+150.1,94 \% e e\right)$ was dissolved in ethanol/water $(5: 1$, total volume $=4.8 \mathrm{~mL})$ by heating to $76{ }^{\circ} \mathrm{C}$ and then the solution was put into $10{ }^{\circ} \mathrm{C}$ refrigerator for $12 \mathrm{~h}$. The crystals were filtered and dried to yield the salt as white crystals $0.443 \mathrm{~g},[\alpha]_{\mathrm{D}}^{20}=+157.1$ (determined by polarimetry, $\left.\mathrm{C}=1, \mathrm{CH}_{3} \mathrm{OH}\right), 99 \%$ ee.

Liberation of (R)-praziquantel amine: The salt racpraziquantel amine-L-tartaric acid (2.725 g, $4.89 \mathrm{mmol})$ was suspended in water $(10 \mathrm{~mL})$ and the $\mathrm{pH}$ of the mixture was adjusted to 12 by adding dropwise $5 \mathrm{~N}$ sodium hydroxide solution. When the salt was completely dissolved, the solution was extracted with dichloromethane $(50 \mathrm{~mL} \times 3)$. The aqueous phase was retained to recover resolving agent. The combined organic layers were washed with brine and concentrated under reduced pressure to give rac-praziquantel amine as white solid $1.513 \mathrm{~g}$ (yield $97 \%$, ee value of rac-praziquantel amine was $99 \%$ determined by HPLC.

Recovery of the resolving agent: The $\mathrm{pH}$ of above aqueous phase was adjusted to 1 by $2 \mathrm{~N}$ hydrochloric acid as soon as possible after the extraction of praziquantel amine. The precipitate was filtered, washed by ice water and dried to yield white product $1.550 \mathrm{~g}$. The potassium chloride was used to saturate the filtration to precipitate the other part of white solid $0.061 \mathrm{~g}$, the combined solid was $1.611 \mathrm{~g}$, yield $94 \%$.

\section{RESULTS AND DISCUSSION}

An efficient approach ${ }^{13}$ was reported to generated racpraziquantel amine, implying a similar availability of that material in quantity when compared to the original Merck process ${ }^{14}$. Then rac-praziquantel amine prepared according to literature ${ }^{13}$ was used as starting material in step 1 (Scheme-I) to form the diastereomeric salt with resolving reagent dibenzoyl-L-tartaric acid in suitable solvent. After recrystallization (step 2), the diastereomeric salt was separated to give R-salt,
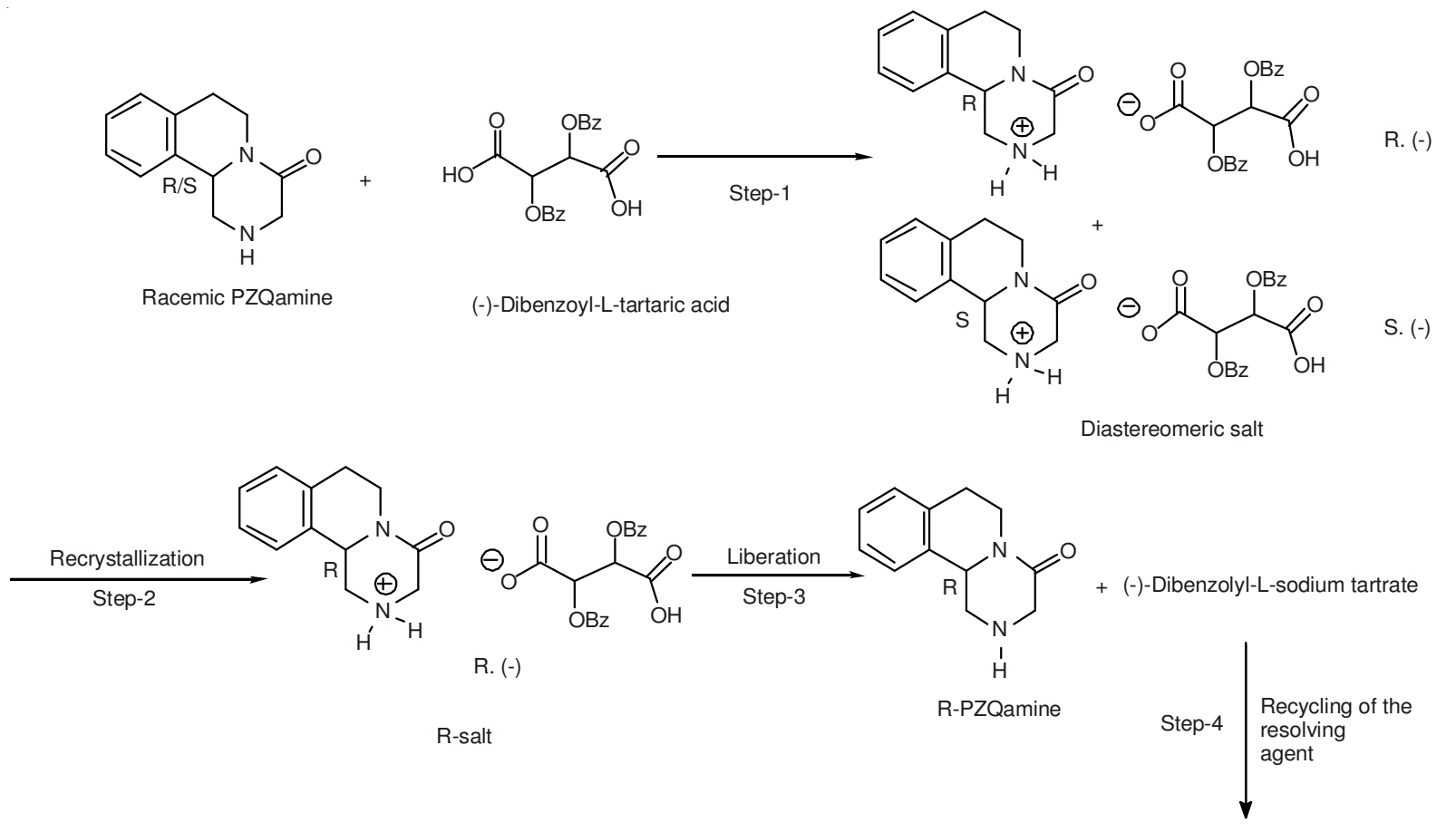

(-)-Dibenzolyl-L-trataric acid

Scheme-I: Resolution procedure of the praziquantel amine 
which was liberated in step 3 to afford $r a c$-praziquantel amine. Finally, the resolving reagent dibenzoyl-L-tartaric acid was recovered.

The yields of both diastereomeric salt in step 1 and R-salt in step 2, the ee value of rac-praziquantel amine and the recovery yield of resolving reagent are absolutely important indicators to evaluate the efficiencies of resolution and are necessary to be optimized to more practical in bulk production.

Few HPLC methods have been reported for the analysis of praziquantel amine. It was mentioned in work of Woelfle et al. ${ }^{12}$ that enantioselective HPLC columns suitable for baseline separation of the enantiomers of praziquantel amine include chiralcel OJ-H (Fig. 1a), Chiralpak IA and AS-H and the eluent solvent system is Heptane: $\mathrm{EtOH}_{2} \mathrm{Et}_{2} \mathrm{NH}(60: 40: 0.2)$ at 0.5$0.7 \mathrm{~mL} / \mathrm{min}$ flow rate, columns found to be unsuitable include Chiralcel OD, OD-H, Chiralpak IB and AD-H. In our studies, three columns (OD-H, OJ-H and AD-H) were investigated (Fig. 1b, 1c, 1d). Actually, the rac-praziquantel amine can be baseline separated much better on AD-H (Fig. 1d) than on OJ-H (Fig. 1c) with optimized eluent system ( $n$-hexane: ethanol:triethylamine $=80: 20: 0.1)$ at flow rate $1 \mathrm{~mL} / \mathrm{min}$. No baseline separation could be reached on OD-H (Fig. 1b). The peak sequence of two isomers of praziquantel amine on $\mathrm{AD}$ $\mathrm{H}$ is the same as that on OJ-H since OJ-H and AD-H are all the positive columns.

It is very necessary to optimize the ratio between $\mathrm{rac}$ praziquantel amine and resolving agent since this ratio plays main influence on both yield of R-salt and ee value of praziquantel amine. It was found that yield of R-salt increased with

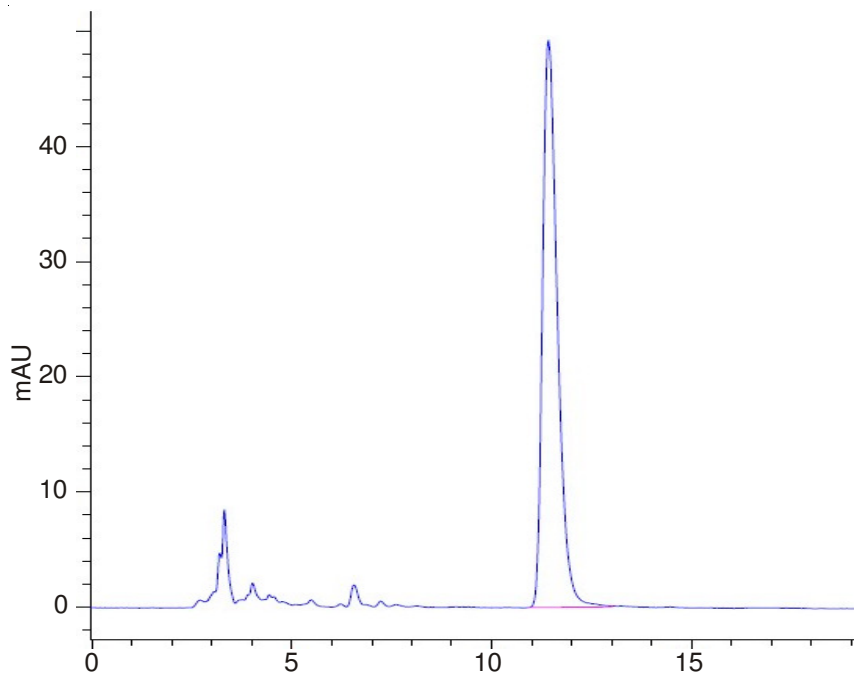

Fig. 1b. HPLC trace for praziquantel amine on OD-H $(4.6 \times 250 \mathrm{~mm}$, DAICEL, eluent system $: n$-hexane:ethanol:triethylamine $=60: 40: 0.1)$

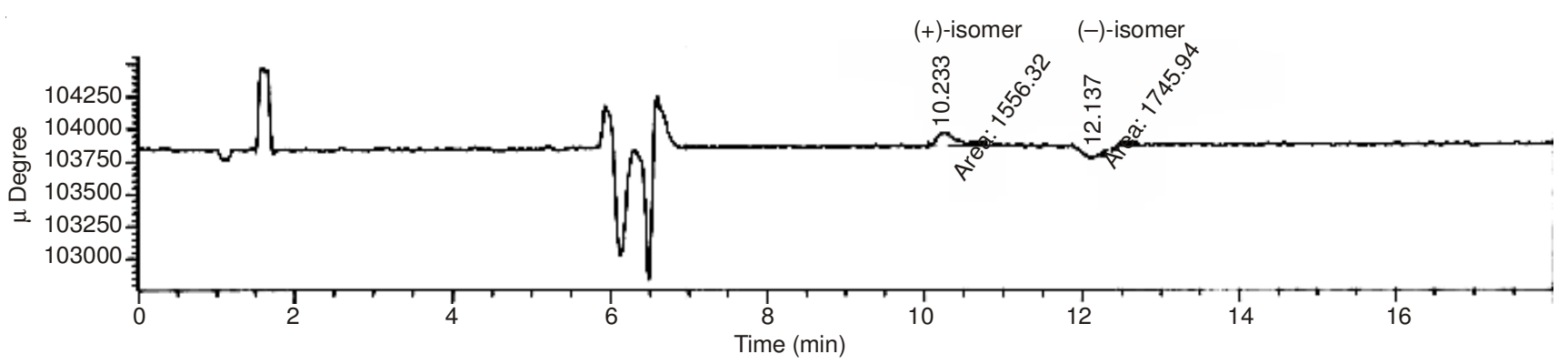

Fig. 1a. HPLC trace for praziquantel amine on OJ-H (Heptane:EtOH = 60:40) [Ref. 12]

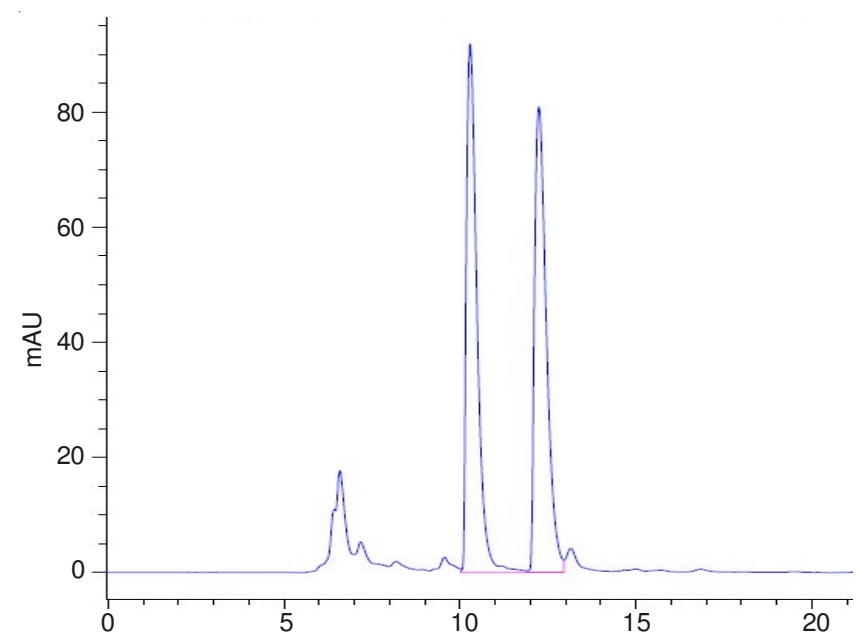

Fig. 1c. HPLC trace for praziquantel amine on OJ-H $(4.6 \times 250 \mathrm{~mm}$, DAICEL, eluent system: $n$-hexane:ethanol:triethylamine $=$ 60:40:0.1)

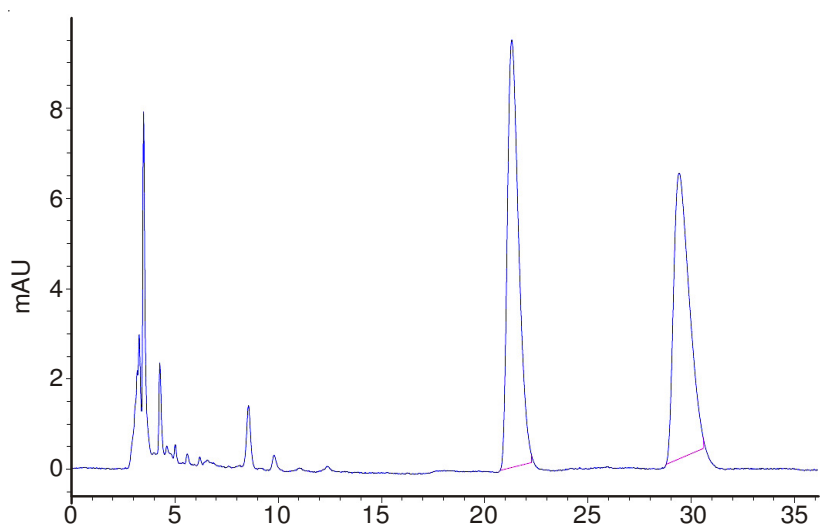

Fig. 1d. HPLC trace for praziquantel amine on AD-H $(4.6 \times 250 \mathrm{~mm}$, DAICEL, eluent system : $n$-hexane:ethanol:triethylamine $=80: 20: 0.1$ )

the improvement of resolving agent equivalent, however thecorresponding ee value of praziquantel amine decreased. The best result was achieved when the ratio was $1: 1$. Considering the cost and the validity, the 1:1 ratio was also the best choice.

Several kinds of mixed solvent system, such as ethanol + water, acetone + water,isopropanol + water were surveyed (Table-1). These solvent systems all could afford $e e$ value over $90 \%$. The mixed solvent of ethanol and water was especially favorable for yield of diastereomeric salt (Table-1, entry 1).

It was found that the ratio between ethanol and water had great effect on yield of salt but less effect on ee value of amine (Table-2). When the total volume was constant, the yield of 


\begin{tabular}{cccc}
\hline \multicolumn{5}{c}{ TABLE-1 } \\
\multicolumn{4}{c}{ VALIDATION OF SOLVENT SYSTEM FOR SALT FORMATION } \\
\hline \multirow{2}{*}{ Entry } & \multirow{2}{*}{ Solvent } & $\begin{array}{c}e e \text { Value of prazi- } \\
\text { quantel amine (\%) }\end{array}$ & $\begin{array}{c}\text { Yield of } \\
\text { R-salt (\%) }\end{array}$ \\
\hline 1 & Ethanol + water & 93 & 90.2 \\
2 & Isopropanol + water & 95 & 82.8 \\
3 & Acetone + water & 94 & 80.4 \\
\hline${ }^{\text {a One equivalent of resolving agent was used }}$
\end{tabular}

the salt increased as the percentage of water was increased to $55.6 \%$ (Table-2, entry 1-7). When percentage of water was enhanced to $61.1 \%$, the resolving agent could be dissolved well and no salt was formed (Table-2, entry 8 ). Therefore, the more volume of reaction solvent was used in order to obtain the good solubility, however the yield of R-salt decreased dramatically (Table-2, entry 9).

The devitrification method affects greatly both yield of salt and $e e$ value of product. After reaction was finished, the reaction solution was stood at $10{ }^{\circ} \mathrm{C}$ immediately. The better yields and $e e$ value of product were obtained for both R-salt (Table-3, entry 3) and S-salt (entry 5) by adding seeding of rac-praziquantel amine-L-tartaric acid and S-praziquantel amine-D-tartaric acid respectively during crystallization process. After seeding addition into the reaction, the salt yield was raised from $43.6 \% 12$ to $50 \%$ (Table-3, entry 3) with ee value being enhanced from 76 to $82 \%$ for R-configuration.

It is difficult to seek out suitable chiral column to evaluate the $e e$ value of the salt in step 1 , because salt formed by hydrogen bond usually can be decomposed on many kinds of column. Therefore, a curve (Fig. 2) indicating the relationship between specific rotation of salt and $e e$ value of praziquantel amine was built before salt recrystallization or liberation since such curve can assess directly the salt quality and guides whether or not the salt needs recrystallization inorder to give high $e e$ value of final product praziquantel amine. The curve was build

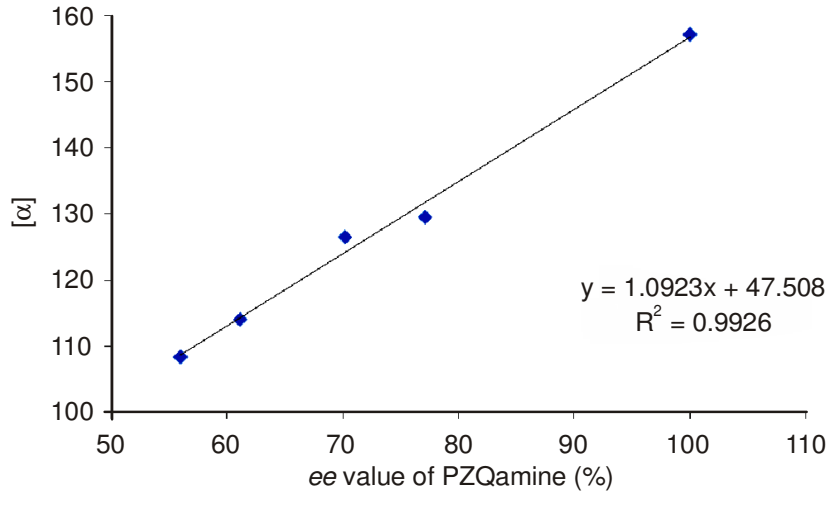

Fig. 2. Relationship between specific rotation of salt and ee value of racpraziquantel amine

up based on several $[\alpha]$ values of salts before recrystallization and the corresponding ee value of praziquantel amine after liberation (Table-4). Actually, the estimated ee values of praziquantel amine by such curve were very close to the ones measured by HPLC (Table-5), which proved the considerably accuracy of the curve.

\begin{tabular}{|c|c|c|}
\hline \multicolumn{3}{|c|}{$\begin{array}{c}\text { TABLE-4 } \\
\text { SPECIFIC ROTATION OF SALT AND CORRESPONDING } \\
e \text { e VALUE OF R- PRAZIQUANTEL AMINE }\end{array}$} \\
\hline Entry & {$[\alpha]^{\mathrm{a}}$} & $\begin{array}{c}e e \text { Value of PZQ } \\
\text { amine }^{\mathrm{b}}\end{array}$ \\
\hline 1 & 108.5 & 56 \\
\hline 2 & 114.1 & 61 \\
\hline 3 & 126.5 & 70 \\
\hline 4 & 129.5 & 77 \\
\hline 5 & 157.1 & 100 \\
\hline
\end{tabular}

${ }^{\mathrm{a}}$ Concentration of salt in $\mathrm{MeOH}$ was $1 \mathrm{~g} / 100 \mathrm{~mL} .{ }^{\mathrm{b}} e e$ Value was determined by chiral HPLC before recrystallization

\begin{tabular}{|c|c|c|c|c|}
\hline \multicolumn{5}{|c|}{ 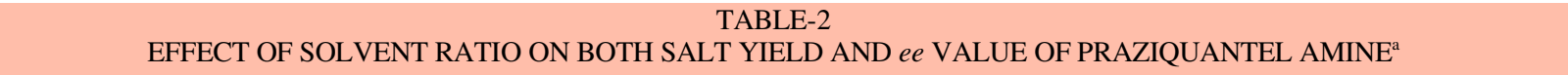 } \\
\hline Entry $^{\mathrm{c}}$ & Water $(\%)$ & Yield of salt (\%) & Yield of R-salt (\%) & $e e$ Value of PZQ amine ${ }^{\mathrm{b}}(\%)$ \\
\hline 1 & 16.6 & 29.5 & 60.1 & 96 \\
\hline 2 & 22.2 & 30.5 & 68.1 & 97 \\
\hline 3 & 27.8 & 35.1 & 72.4 & 94 \\
\hline 4 & 33.3 & 37.7 & 75.3 & 92 \\
\hline 5 & 38.9 & 38.8 & 76.6 & 94 \\
\hline 6 & 50.0 & 40.1 & 80.7 & 91 \\
\hline 7 & 55.6 & 41.6 & 39.4 & 94 \\
\hline $8 \mathrm{~d}$ & 61.1 & - & - & - \\
\hline $9 \mathrm{e}$ & 61.1 & 8.23 & 16.2 & 97 \\
\hline
\end{tabular}

${ }^{\mathrm{a} O n e}$ equivalent of resolving agent was used. The mixed solvent composed of ethanol and water. ${ }^{\mathrm{b}}$ The $e e$ value was determined by HPLC before recrystallization. ${ }^{\mathrm{c}}$ The total amount of solvent was the same for entry $1-8 .{ }^{\mathrm{d}}$ The resolving agent couldn't be dissolved completely. ${ }^{\mathrm{d}}$ The total amount of solvent was increased so that the resolving agent was dissolved

\begin{tabular}{|c|c|c|c|c|c|}
\hline \multicolumn{6}{|c|}{$\begin{array}{c}\text { TABLE-3 } \\
\text { VALIDATION OF DEVITRIFICATION METHOD }\end{array}$} \\
\hline $\begin{array}{l}\text { Configuration of } \\
\text { amine }\end{array}$ & Entry & Condition & $\begin{array}{l}\text { Yield of salt } \\
(\%)\end{array}$ & $\begin{array}{l}\text { Yield of R/S-salt } \\
(\%)\end{array}$ & $\begin{array}{l}\text { ee Value of praziquantel } \\
\text { qamine } \mathrm{c}^{\mathrm{c}}(\%)\end{array}$ \\
\hline \multirow{3}{*}{$\mathrm{R}$} & 1 [Ref. 12] & Without seeding & 43.6 & 76.8 & 76 \\
\hline & 2 & Without seeding & 48.9 & 86.1 & 76 \\
\hline & 3 & With seeding & 50.0 & 91.0 & 82 \\
\hline \multirow{2}{*}{$\mathrm{S}$} & 4 & Without seeding & 37.3 & 72.4 & 94 \\
\hline & 5 & With seeding & 43.7 & 83.8 & 94 \\
\hline
\end{tabular}

${ }^{\mathrm{a}}$ One equivalent of resolving agent was used. The percentage of water is $55.6 \%{ }^{\mathrm{b}}$ The yield was based on half of rac-PZQamine. ${ }^{\mathrm{C}}$ The $e e$ value was determined by HPLC before recrystallization 
TABLE-5

EFFECT OF RECRYSTALLIZATION ON $e e$ AND $[\alpha]$ VALUES

\begin{tabular}{|c|c|c|c|c|c|}
\hline \multirow{2}{*}{ Target product } & \multirow{2}{*}{ Entry } & \multirow{2}{*}[\alpha]{$^{\mathrm{a} /[\alpha]^{\mathrm{b}}}$} & $e e$ Value of amine $e^{c}$ & $e e$ Value of amine ${ }^{\mathrm{d}}$ & \multirow{2}{*}{$\operatorname{Loss}^{\mathrm{e}}(\%)$} \\
\hline & & & $e e^{\mathrm{a}} / e e^{\mathrm{b}}$ & $e e^{\mathrm{a}} / e e^{\mathrm{b}}$ & \\
\hline \multirow{4}{*}{ R- PZQamine } & 1 & $132.9 / 155.9$ & $78 / 99$ & $77 / 99$ & 8.0 \\
\hline & 2 & 131.2 / 156.1 & $77 / 99$ & $76 / 99$ & 8.0 \\
\hline & 3 & $137.1 / 157.0$ & $82 / 99$ & $82 / 99$ & 10.0 \\
\hline & 4 & $138.2 / 156.3$ & $83 / 99$ & $82 / 99$ & 10.0 \\
\hline \multirow{2}{*}{ S- PZQamine } & 5 & $149.6 / 158.0$ & $94 / 99$ & $93 / 99$ & 8.6 \\
\hline & 6 & $149.7 / 156.1$ & 94 / 99 & $93 / 99$ & 8.6 \\
\hline
\end{tabular}

${ }^{\mathrm{a}}[\alpha]$ value was determined before recrystallization. ${ }^{\mathrm{b}}[\alpha]$ value was determined after recrystallization. ${ }^{\mathrm{c}} e e$ value was estimated through curve in Fig. 2. ${ }^{\mathrm{d}} e e$ value was measured by HPLC. ${ }^{\mathrm{e}}$ Loss of R-(-)-PZQ amine or S-(-)-PZQ amine in recrystallization processes

Being supervised by above curve (Fig. 2), when the $[\alpha]$ value of salt was achieved over 156 , the $e e$ value of praziquantel amine could be raised over $99 \%$. After one time of recrystallization, the $[\alpha]$ value of salts were achieved over 156 , the ee value of praziquantel amines were reached to $99 \%$. However, $8-10 \%$ of product was lost in recrystallization process.

Liberation of praziquantel amine in step 3: To avoid racemization, the liberation of praziquantel amine was conducted in ice-water bath when $\mathrm{NaOH}$ solution was added into salt. The $e e$ value was obtained over $99 \%$, which indicated that racemization didn't happen in liberation procedure. On the other hand, due to the water-solubility of praziquantel amine, the salt was suspended in as less water as possible, which was adjusted $\mathrm{pH}$ further with considerable concentrated $\mathrm{NaOH}$ aqueous solution. In this way, yields of praziquantel amine were stabilized between 94-97\%.

Recovering of the resolving agent: The above basic water phase after extraction of praziquantel amine was adjusted to $\mathrm{pH}=1-2$ to precipitate resolving agent. In view of water-solubility of dibenzoyl tartaric acid, the water phase was saturated by salt (such as $\mathrm{NaCl}, \mathrm{KCl}$ ) so that the resolving reagent was precipitated very well. After precipitation,the resolving reagent was collected by simple filtration and the recovery yield of dibenzoyl tartaric acid was reached $94 \%$.

\section{Conclusion}

A suitable solvent system and an effective devitrification method with seeding addition were chosen and validated for salt formation. The reaction conditions were optimized to achieve both good yields of salt, praziquantel amine, recovery of resolving agent and high ee value of praziquantel amine. This optimized process of resolving praziquantel amine enables a practical and economical bulk synthesis and promises to make it easy to match industrial production.

\section{ACKNOWLEDGEMENTS}

The authors are grateful to the National High Technology Research and Development Program of China (863 Program, Project number: 2012AA020306) for the financial support.

\section{REFERENCES}

1. L. Chitsulo, D. Engels, A. Montresor and L. Savioli, Acta Trop., 77, 41 (2000).

2. M.J. Doenhoff, D. Cioli and J. Utzinger, Curr. Opin. Infect. Dis., 21, 659 (2008).

3. X. Shu-Hua and B.A. Catto, J. Infect. Dis., 159, 589 (1989).

4. M.H. Wu, C.C. Wei, Z.Y. Xu, H.C. Yuan, W.N. Lian, Q.-J. Yang, M. Chen, Q.-W. Jiang, C.-Z. Wang, S.-J. Zhang, Z.-D. Liu, R.-M. Wei, S.J. Yuan, L.-S. Hu and Z.-S. Wu, Am. J. Trop. Med. Hyg., 45, 345 (1991).

5. Y.H. Liu, M.X. Qian, X.G. Wang, J. Jia, Q.N. Wang, Y.F. Jiang, R.Q. Wang, S.H. Yan, B.Y. Chen and J.S. Li, Chin. Med. J. (Engl.), 99, 935 (1986).

6. P. Roszkowski, J.K. Maurin and Z. Czarnocki, Tetrahedron Asymm., 17, 1415 (2006).

7. T. Meyer, H. Sekljic, S. Fuchs, H. Bothe, D. Schollmeyer and C. Miculka, PLoS Negl. Trop., 3, e357 (2009).

8. M.J. Doenhoff and L. Pica-Mattoccia, Anti Infect.Ther., 4, 199 (2006).

9. C.R. Caffrey, Curr. Opin. Chem. Biol., 11, 433 (2007).

10. A. Fenwick and J.P. Webster, Curr. Opin. Infect. Dis., 19, 577 (2006).

11. Drug Development and Evaluation for Helminths and Other Neglected Tropical Diseases, Business Plan 2008-2013, WHO/TDR (2007).

12. M. Woelfle, J.-P. Seerden, J. de Gooijer, K. Pouwer, P. Olliaro and M.H. Todd, PLoS Negl. Trop. Dis., 5, e1260 (2011).

13. (a) J.H. Kim, Y.S. Lee, H. Park and C.S. Kim, Tetrahedron, 54, 7395 (1998); (b) C.H. Yang, L.Z. Zhang, Y. Zheng and D.Q. Sun, Asian J. Chem., 25, 9415 (2013).

14. P. Andrews, H. Thomas, R. Pohlke and J. Seubert, Praziquantel. Med. Res. Rev., 3, 147 (1983). 